\title{
Numerical Dissipation Control in High Order Shock-Capturing Schemes for LES of Low Speed Flows
}

\author{
D. V. Kotov, H. C. Yee, A. A. Wray, B. Sjögreen and A. G. Kritsuk
}

\begin{abstract}
The Yee \& Sjögreen adaptive numerical dissipation control in high order scheme (High Order Filter Methods for Wide Range of Compressible Flow Speeds, ICOSAHOM 09, 2009) is further improved for DNS and LES of shock-free turbulence and low speed turbulence with shocklets. There are vastly different requirements in the minimization of numerical dissipation for accurate turbulence simulations of different compressible flow types and flow speeds. Traditionally, the method of choice for shock-free turbulence and low speed turbulence are by spectral, high order central or high order compact schemes with high order linear filters. With a proper control of a local flow sensor, appropriate amount of numerical dissipation in high order shock-capturing schemes can have spectral-like accuracy for compressible low speed turbulence flows. The development of the method includes an adaptive flow sensor with automatic selection on the amount of numerical dissipation needed at each flow location for more accurate DNS and LES simulations with less tuning of parameters for flows with a wide range of flow speed regime during the time-accurate evolution, e.g., time varying random forcing. An automatic selection of the different flow sensors catered to the different flow types is constructed. A
\end{abstract}

D. Kotov

Bay Area Environmental Research Institute, 625 2nd St. Ste 209 Petaluma, CA 94952 e-mail: dmitry.v.kotov@nasa.gov

Work done while the first author was a postdoctoral fellow at the Center for Turbulence Research, Stanford University

H. C. Yee

NASA Ames Research Center, Moffett Field, CA 94035 e-mail: helen.m.yee@nasa.gov

A. Wray

NASA Ames Research Center, Moffett Field, CA 94035 e-mail: Alan.A.Wray@nasa.gov

B. Sjögreen

Lawrence Livermore National Laboratory, Box 808, L-422, Livermore, CA 94551-0808 e-mail: sjogreen2@1lnl.gov

A. G. Kritsuk, University of California, San Diego, La Jolla, CA, 92093-0424 e-mail: akritsuk@ucsd.edu 
Mach curve and high-frequency oscillation indicators are used to reduce the tuning of parameters in controlling the amount of shock-capturing numerical dissipation to be employed for shock-free turbulence, low speed turbulence and turbulence with strong shocks. In Kotov et al. (High Order Numerical Methods for LES of Turbulent Flows with Shocks, ICCFD8, Chengdu, Sichuan, China, July 14-18, 2014) the LES of a turbulent flow with a strong shock by the Yee \& Sjögreen scheme indicated a good agreement with the filtered DNS data. A work in progress for the application of the adaptive flow sensor for compressible turbulence with time-varying random forcing is forthcoming. The present study examines the versatility of the Yee \& Sjögreen scheme for DNS and LES of traditional low speed flows without forcing. Special attention is focused on the accuracy performance of this scheme using the Smagorinsky and the Germano-Lilly SGS models.

\section{Introduction and Objective}

For the last decade, high order shock-capturing methods with numerical dissipation controls have been the state-of-the-art numerical approach for direct numerical simulation (DNS) and large eddy simulation (LES) of turbulent flows with shocks. See, for example, $[1,2,3,4,5,6,7,8,9,10,11]$. Also see examples of recently developed implicit LES (ILES) methods and comparison of the performance of different numerical schemes (mostly are second to fifth orders) in [12, 13, 14, 15]. The majority of these methods involve flow sensors with parameter tuning applied depending on the flow type. Some of the flow sensors were designed for certain flow types and might not preserve their high accuracy when used to simulate a different flow type. In a study presented in Johnsen et al. [9], all of the shock-capturing schemes involve tuning of the parameters. It appears that the Yee \& Sjögreen filter scheme is not as accurate as the hybrid scheme presented in [9] as the key parameter $\kappa$ responsible for minimizing the numerical dissipation in the 2007 Yee \& Sjögreen scheme [2] was mandated to be the same for all considered test case reported in [9]. See [1, 6] for a description of better control of numerical dissipation using a local $\kappa$. The hybrid scheme presented in [9] which employed the Ducros et al. flow sensor [16] also consists of a key tuning parameter $\delta$. From our study presented below of the same Taylor-Green vortex problem considered in [9], the cut-off parameter $\delta$ to be 1 to achieve the best accurate result. On the other hand, for the isotropic turbulence with shocklets test case, the Ducros et al. flow sensor $\delta$ parameter has to be reduced, mostly by trial and error. Yet in another study [7] for turbulence interacting with a high speed stationary shock, depending on the Mach number and turbulent Mach number, different $\delta$ are required for each case.

In recognizing the different requirements on numerical dissipation control for DNS and LES of a variety of compressible flow types, Yee \& Sjogreen, [1], presented a general framework for a local $\kappa$ and the accompanying variety of flow sensors were introduced into their high order nonlinear filter scheme. Aside from suggesting different local $\kappa$ formulation, Yee \& Sjogreen also proposed the use of a 
combination of different flow sensors. See the next section for a complete description of the local $\kappa$ formulation. Their proposed scheme with numerical dissipation control has not been studied extensively until recently. A subset to the sequel to [1] was presented in $[17,6]$. This is yet another sequel to Yee \& Sjogreen.

The goal of this work is to examine the different combinations of flow sensors for DNS and LES of low speed turbulent flows. For the DNS and LES numerical experiments, two low speed flows are considered: (a) The 3D compressible viscous counterpart of the very low speed shock-free turbulence Taylor-Green vortex problem considered in [9] and (b) the same isotropic turbulence with shocklets as in [9]. A work in progress for the application of the adaptive flow sensor for compressible turbulence with time-varying random forcing is forthcoming. A preliminary result is included in the Appendix.

\section{High Order Nonlinear Filter Schemes}

This section gives a brief overview of the high-order nonlinear filter scheme of Yee et al. $[2,1,4,6]$ for accurate computations of DNS and LES of compressible turbulence for a wide range of flow types. Within the confines of the considered integrated approach, this method attempts to introduce as little numerical dissipation as possible. The basic design principle of the filter scheme is to minimize the use of numerical dissipation inherited from standard high order shock-capturing schemes that were developed for rapidly developing unsteady shock flows. Without any modification or redesigning of these standard schemes, they are too diffusive for DNS and LES computations. The nonlinear filter scheme consists of three steps, as described in the following three subsections.

\subsection{Preprocessing Step}

Before the application of a high-order non-dissipative spatial base scheme, a preprocessing step is employed to improve numerical stability. The inviscid flux derivatives of the governing equations are split into the following three ways, depending on the flow types and the desire for rigorous mathematical analysis or physical argument.

- Entropy splitting of [18] and [19, 20]. This splitting of the inviscid flux derivative into two parts is non-conservative and the derivation is based on entropy variables and energy norm stability for the compressible Euler equations with boundary closure for the initial boundary value problem.

- The Ducros et al. splitting [21] for systems. This is a conservative splitting and the derivation is based on physical arguments.

- Tadmor entropy conservation formulation for systems [22]. The derivation is based on mathematical analysis. It is a generalization of Tadmor's entropy formulation to systems and has only been tested on a limited number of test cases. The 
findings are that the Tadmor entropy conservation formulation is more diffusive than the other two splittings.

\subsection{Base Scheme Step}

A full time step is advanced using a high-order non-dissipative (or very low dissipation) spatially central scheme on the split form of the governing partial differential equations (PDEs) (i.e., after the preprocessing step). A summation-by-parts (SBP) boundary operator $[23,24]$ and matching order conservative high-order free stream metric evaluation for curvilinear grids [25] are used. Note that the base scheme can be a high order compact scheme [26], the standard high order central schemes or spectral methods. However the same entropy stable SBP boundary closure for high order central schemes is not valid for the latter base schemes.

High-order temporal discretization such as the third-order or fourth-order RungeKutta (RK3 or RK4) method is used. It is remarked that other temporal discretizations can be used for the base scheme step.

\subsection{Post-Processing (Nonlinear Filter Step)}

To further improve the accuracy of the computed solution from the base scheme step, after a full time step of a non-dissipative high-order spatial base scheme on the split form of the governing equation(s), the post-processing step is used to nonlinearly filter the solution by a dissipative portion of a high-order shock-capturing scheme with a local flow sensor. The flow sensor provides locations and amounts of built-in shock-capturing dissipation that can be further reduced or eliminated. At each grid point a local flow sensor is employed to analyze the regularity of the computed flow data. Only the strong discontinuity locations would receive the full amount of shock-capturing dissipation. In smooth regions no shock-capturing dissipation would be added, unless high frequency oscillations are developed, owning to the possibility of numerical instability in long time integrations of nonlinear governing PDEs. In regions with strong turbulence, if needed, a small fraction of the shock-capturing dissipation would be added to improve stability.

Note that the filter numerical fluxes only involve the inviscid flux derivatives regardless if the flow is viscous or inviscid. If viscous terms are present, a matching high order central difference operator (as the inviscid difference operator) is included on the base scheme step. For ease of SBP numerical boundary closure for the viscous flux derivatives, the same inviscid central difference operator for the first derivative is employed twice for the viscous flux derivatives.

For simplicity of presentation, considered the 3D Euler equations

$$
\frac{\partial U}{\partial x}+\frac{\partial E}{\partial x}+\frac{\partial F}{\partial x}+\frac{\partial G}{\partial x}=0
$$


where $E, F$ and $G$ are inviscid flux derivatives in the $x, y$ and $z$ directions, respectively.

Let $U^{*}$ be the solution after the completion of the full time step of the base scheme step. The final update of the solution after the filter step is (with the numerical fluxes in the $y$-and $z$-directions as well as their corresponding $y$ - and $z$-direction indices on the $x$ inviscid flux omitted)

$$
U_{j, k, l}^{n+1}=U_{j, k, l}^{*}-\frac{\Delta t}{\Delta x}\left[H_{j+1 / 2}^{*}-H_{j-1 / 2}^{*}\right], \quad H_{j+1 / 2}^{*}=R_{j+1 / 2} \bar{H}_{j+1 / 2},
$$

where $R_{j+1 / 2}$ is the matrix of right eigenvectors of the Jacobian of the inviscid flux vector in terms of Roe's average states based on $U^{*} . H_{j+1 / 2}^{*}$ and $H_{j-1 / 2}^{*}$ are "filter" numerical fluxes in terms of Roe's average states based on $U^{*}$. Denote the elements of the filter numerical flux vector $\bar{H}_{j+1 / 2}$ by $\bar{h}_{j+1 / 2}^{l}, l=1,2, \ldots, 5$, where $\bar{h}_{j+1 / 2}^{l}$ has the form

$$
\bar{h}_{j+1 / 2}^{l}=\frac{\kappa_{j+1 / 2}^{l}}{2} w_{j+1 / 2}^{l} \phi_{j+1 / 2}^{l} .
$$

Here $w_{j+1 / 2}^{l}$ is a flow sensor to activate the nonlinear numerical dissipation portion of a high order shock-capturing scheme $\frac{1}{2} \phi_{j+1 / 2}^{l}$, and $\kappa_{j+1 / 2}^{l}$ is a positive flow dependent parameter that is less than or equal to one to control the amount of shock-capturing dissipation to be used. The nonlinear dissipative portion of a highresolution shock-capturing scheme $" \frac{1}{2} \phi_{j+1 / 2}^{l}$ " can be any shock-capturing scheme. The choice of the parameter $\kappa_{j+1 / 2}^{l}$ can be different for different flow types and is automatically chosen by using the local $\kappa_{j+1 / 2}^{l}$ described in [1]. The flow sensor $w_{j+1 / 2}^{l}$ can be a variety of formulae introduced in the literature or can be switched from one flow sensor to another, depending on the computed flow data at that particular location. For a variety of local flow sensors with automatic selection of the proper parameter, depending on different flow type, see [1]. The form of TauberSandham [10] for the filter numerical flux uses the Ducros et al. flow sensor as $\kappa_{j+1 / 2}^{l}$ and the Harten artificial compression method formula (ACM) as the flow sensor indicated in [4] and similarly in [11] are part of the Yee \& Sjögreen adaptive numerical dissipation control generalization filter formulae. For the numerical experiments presented, we mainly concentrate on the wavelet flow sensor of Yee \& Sjogreen, the Ducros et al. flow sensor [16] and the artificial compression method flow sensor of [4]. For the wavelets and ACM flow sensors, see the aforementioned references cited. The Ducros et al. flow sensor designed mainly to capture flows containing shocks and vorticity has the form:

$$
w=\frac{(\nabla \cdot \mathbf{u})^{2}}{(\nabla \cdot \mathbf{u})^{2}+\omega^{2}+\varepsilon} .
$$

Here $\mathbf{u}$ is the velocity vector, $\omega$ is the vorticity magnitude and $\varepsilon$ is a small number to avoid division by zero (e.g., $10^{-6}$ ). The Ducros et al. flow sensor consists of a cut off 
parameter $\delta$ that can be used to switch on or off the dissipative portion of the high order shock-capturing scheme. If $\delta$ is set to be one, the dissipation only switches on when it encounters a shock wave. For lower value of the cut off $\delta$ parameter, vorticity can be detected. The next section discuss the low Mach number kappa curve developed in [1].

\subsubsection{An efficient global $\kappa$ for low Mach number and smooth flows:}

The flow speed indicator formula of $\mathrm{Li} \& \mathrm{Gu}$ to overcome the shortcomings of "low speed Roe scheme" [27] was modified to obtain an improved global $\kappa_{j+1 / 2}^{l}$ denoted by $\bar{\kappa}$ for (3) to minimize the tuning of the original $\kappa$ for low Mach number flows. $\bar{\kappa}$ has the form:

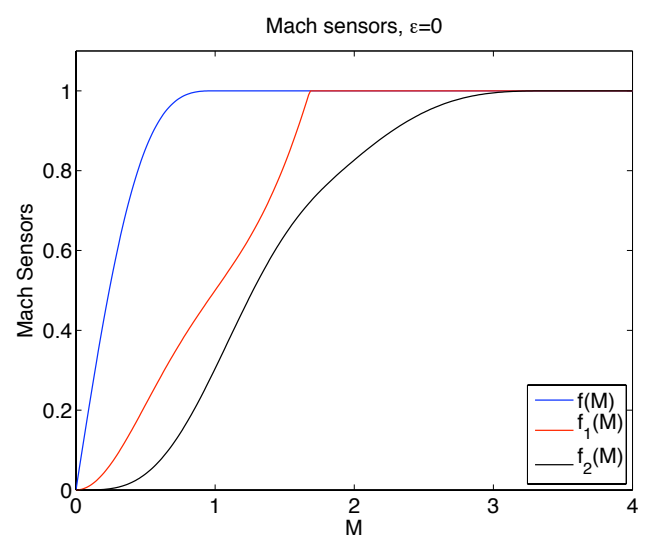

Fig. 1 Mach number sensors. $f(M)$ (blue) function by Li and Gu, $f_{1}(M)$ (red) modified $f(M)$, and $f_{2}(M)$ (black) (includes low supersonic Mach numbers).

$$
\bar{\kappa}=f_{1}(M) \kappa,
$$

with

$$
f_{1}(M)=\min \left(\frac{M^{2}}{2} \frac{\sqrt{4+\left(1-M^{2}\right)^{2}}}{1+M^{2}}, 1\right) .
$$

Here $M$ is the maximum Mach number of the entire computational domain at each stage of the time evolution. $f_{1}(M)$ has the same form as [27] except there is an extra factor " $\frac{M}{2}$ " added to the first argument on the right-hand-side of the original form $f(M)$ in equation (18) of [27]. The added factor provides a similar value of the tuning $\kappa$ observed from numerical experimentation reported in aforementioned cited references. With the flow speed indicator $f_{1}(M)$ in front of $\kappa$, the same $\kappa$ used for the supersonic shock problem can be used without any tuning for the very low 
speed turbulent flow cases. Another minor modification of the above is,

$$
\overline{f_{1}(M)}=\max \left(\min \left(\frac{M^{2}}{2} \frac{\sqrt{4+\left(1-M^{2}\right)^{2}}}{1+M^{2}}, 1\right), \varepsilon\right),
$$

where $\varepsilon$ is a small threshold value to avoid completely switching off the dissipation. A function which retains the majority of $f_{1}(M)$ but includes larger Mach number for not very strong shocks is

$$
f_{2}(M)=(Q(M, 2)+Q(M, 3.5)) / 2
$$

or

$$
\overline{f_{2}(M)}=\max ((Q(M, 2)+Q(M, 3.5)) / 2, \varepsilon),
$$

where

$$
Q(M, a)=\left\{\begin{array}{ll}
P(M / a) & M<a \\
1 & \text { otherwise }
\end{array} .\right.
$$

The polynomial

$$
P(x)=x^{4}\left(35-84 x+70 x^{2}-20 x^{3}\right)
$$

is monotonically increasing from $P(0)=0$ to $P(1)=1$ and has the property that $P^{\prime}(x)$ has three continuous derivatives at $x=0$ and at $x=1$.

Below supersonic speeds, a simple and efficient global $\bar{\kappa}$ can be obtained according to the maximum Mach number of the entire flow field and the value is determined by $f_{1}(M)$ or $f_{2}(M)$ for non-zero $\omega_{j+1 / 2}^{l}$. It is noted that if the original $f(M)$ were used instead of $f_{1}(M)$ or $f_{2}(M)$ in Eq.(5), the amount of nonlinear filter dissipation could be too large for very low speed turbulent flows (for the same fixed $\kappa)$. See Fig. 1 for details.

\subsubsection{Local flow sensor for a wide spectrum of flow speed and shock strength}

At each time step and grid point, the aforementioned global $\bar{\kappa}$ is not sufficient to reduce the amount of numerical dissipation where needed for flows that contains a variety of flow features. A more appropriate approach is to obtain a "local $\kappa$ " that is determined according to the above at each grid point. If known, a dominating shock jump variable should be used for shock detections. In other words, the filter numerical flux indicated in Eq.(3) is now include $\bar{\kappa}$ as part of the local kappa formulation.

In the case of unknown physics and without experimental data or theory for comparison, $\kappa_{j+1 / 2}^{l}$ has to depend on the local Mach number in low speed or smooth flow regions, depend on local shock strength in shock regions and depend on turbulent fluctuations in vortical regions in order to minimize the tuning of parameters. According to the flow type locally, for each non-zero wavelet indicator $\omega_{j+1 / 2}^{l}, \kappa_{j+1 / 2}^{l}$ should provide the aforementioned amount (between $(0,1)$ ) to be filtered by the shock-capturing dissipation $\phi_{j+1 / 2}^{l}$. For problems containing turbulence and strong 
shocks, the shock strength should come into play. One measure of the shock strength can be based on the numerical Schlieren formula [28] for the chosen variables that exhibit the strongest shock strength. In the vicinity of turbulent fluctuation locations, $\kappa_{j+1 / 2}^{l}$ will be kept to the same order as in the nearly incompressible case, except in the vicinity of high shear and shocklets.

Due to the fact that $\bar{\kappa}$ works well for local Mach number below $0.4, \kappa$ only needs to be modified in regions that are above 0.4 . In other words, the final value of $\kappa_{j+1 / 2}^{l}$ is determined by the previous local $\bar{\kappa}$, if the local Mach number is below 0.4. If the local Mach number is above 0.4 , at discontinuities detected by the non-zero wavelet indicator $\omega_{j+1 / 2}^{l}, \kappa_{j+1 / 2}^{l}$ is determined by the shock strength (normalized between $(0,1))$ based on the Schlieren formula near discontinuities. Again, if known, dominating shock jump variables should be used for shock detections. At locations with turbulence, determined by the turbulent sensor (e.g., $\omega_{j+1 / 2}^{l}$ obtained from employing wavelets with higher order vanishing moments), $\kappa_{j+1 / 2}^{l}$ is kept to the same order as in the nearly incompressible case, except in the vicinity of high shear and shocklet locations, where a slightly larger $\kappa_{j+1 / 2}^{l}$ would be used. Methods in detecting turbulent flow can be (a) Wavelets with higher order vanishing moments, and (b) Wavelet based Coherent Vortex Extraction (CVE) of Farge et al. [29] (Split the flow into two parts: Active coherent vortices and incoherent background flow).

It is noted that the nonlinear filter step described above should not be confused with the LES filtering operation. For previous studies on the performance of this filter scheme in DNS and LES simulations, see [4, 30, 3, 31, 5, 32, 1, 6]. This scheme has been validated for DNS of a 3D channel flow, 2D temporal and spatial evolving mixing layers, Richtmyer-Meshkov instability, 3D Taylor-Green vortex, 3D isotropic turbulence with shocklets, extreme condition flows, and a 3D supersonic LES of temporal evolving mixing layers comparing with experimental data.

The current numerical experimental study is confined to the following four forms for the filter numerical flux in conjunction with DNS grid refinement study and two LES models denoted by LES1 and LES2 (to be discussed in the next subsection). For certain low speed turbulence flows, the schemes of choice are spectral and high order compact or central schemes with SBP boundary closures. The nonlinear filter step is not needed and this option using the high order central scheme base scheme only is indicated on the last bullet.

- The first form of the filter numerical flux indicated in [1] is where $\kappa_{j+1 / 2}^{l}$ is the Mach curve for low speed flow described in [1]. $w_{j+1 / 2}$ is the wavelet flow sensor. If the tenth-order central base scheme, entropy splitting and the dissipative portion of the ninth-order WENO scheme (WENO9) are employed, it is denoted by WENO9fi-Esplit-Wav $\kappa(i)$. If the Ducros et al. splitting is used, it is denoted by WENO9fi-Dsplit-Wav.

- The second form of the numerical flux indicated in [1] is where $\kappa_{j+1 / 2}^{l}$ is a constant based on the initial Mach number of the flow, and $w_{j+1 / 2}$ is the wavelet flow sensor. If the tenth-order central base scheme, entropy splitting and the dissipative portion of WENO9 are employed, it is denoted by WENO9fi-Esplit- 
Wav $\kappa=$ const. Similarly if the Ducros et al. splitting is used, it is denoted by WENO9fi-Dsplit-Wav $\kappa=$ const.

- The third form of the numerical flux is where $\kappa_{j+1 / 2}^{l}$ is a positive non-zero constant, and $w_{j+1 / 2}$ is the Ducros et al. flow sensor in conjunction with the $\delta$ cut off parameter. If the same base scheme and the same dissipative portion of WENO9 are used, they are denoted by WENO9fi-Esplit-Ducr \& WENO9fi-Dsplit-Ducr.

- The fourth form of the numerical flux is where the Ducros et al. flow sensor is used as $\kappa_{j+1 / 2}^{l}$, and $w_{j+1 / 2}$ is the wavelet flow sensor or the ACM flow sensor. For the same base scheme and the dissipative portion of WENO9, it is denoted by WENO9fi-Esplit-WavD \& WENO9fi-Dsplit-WavD (WENO9fi-Esplit-AcmD \& WENO9fi-Dsplit-AcmD).

- The last form is when no nonlinear filter step is used, i.e., only the base scheme step is employed. It is denoted by C10-Esplit in the case of employing the tenthorder central base scheme with entropy splitting. If the Ducros et al. splitting is used, it is denoted by C10-Dsplit. In the case when no splitting is used and the 12th-order linear dissipation is added to the base scheme for stability, the method is denoted by C10-AV12.

The aforementioned nonlinear filtered scheme options is employed for the DNS computations of the full 3D Euler and Navier-Stokes equations first. Then the same scheme will be employed for the filtered Navier-Stokes equations for two LES SGS models. These LES SGS models will be presented on the next subsection. The forms (1) - (4) for the filter numerical flux were chosen to demonstrate that for low speed turbulence flows without strong shear waves, the constant $\kappa$ vs. the local $\kappa_{j+1 / 2}^{l}$ behave similarly. The main difference when using the constant $\kappa$ parameter is that one has to know the flow structure of the entire evolution a priori in order to select the proper constant $\kappa$ parameter.

Preliminary study showed that using the ACM flow sensor in conjunction with the Ducros et al. as $\kappa_{j+1 / 2}^{l}$ is more diffusive than the rest of the above considered options and its results are not included here. All of the results shown use the thirdorder Runge-Kutta temporal discretization. The results obtained using the fourthorder Runge-Kutta discretization are similar.

\section{Governing Equations \& SGS Models}

We consider the governing equations written in the following form

$$
\begin{aligned}
\partial_{t} \bar{\rho}+\partial_{j}\left(\bar{\rho} \tilde{u}_{j}\right) & =0 \\
\partial_{t}\left(\bar{\rho} \tilde{u}_{i}\right)+\partial_{j}\left(\bar{\rho} \tilde{u_{i}} \tilde{u}_{j}+\bar{p} \delta_{i j}-\check{\tau}_{i j}+\tau_{i j}^{S}\right) & =0 \\
\partial_{t}(\bar{\rho} \tilde{E})+\partial_{j}\left(\bar{\rho} \tilde{E} \tilde{u}_{j}+\bar{p} \tilde{u}_{j}-\check{\tau}_{i j} \tilde{u}_{i}+\check{q}_{j}+q_{j}^{S}\right) & =0,
\end{aligned}
$$

where $\rho$ is density, $u_{i}$ is $i^{\text {th }}$ velocity component, $p$ is pressure, $T$ is the temperature, $E$ is the total Energy, and $t$ is time. For given $f$, LES filtering operation is denoted 
as $\bar{f}$. Favre filtering operation is denoted as $\tilde{f}=\overline{\rho f} / \bar{\rho}$, and $\breve{f}$ stands for the function of Favre-filtered variables:

$$
\check{\tau}_{i j}=2 \mu(\tilde{T})\left(\tilde{S}_{i j}-\frac{1}{3} \delta_{i j} \partial_{k} \tilde{u}_{k}\right), \quad \tilde{S}_{i j}=\left(\partial_{j} \tilde{u}_{i}+\partial_{i} \tilde{u}_{j}\right) / 2, \quad \check{q}_{j}=-\lambda(\tilde{T}) \partial_{j} \tilde{T},
$$

where dynamic viscosity is given by $\mu(T)=\mu_{0}\left(T / T_{0}\right)^{3 / 4}$ and thermal conductivity is expressed through a constant Prandtl number $P r$ and heat capacity at constant pressure $c_{p}\left(\lambda(T)=c_{p} \mu(T) / P r\right)$. The equation of state is $\bar{p}=R \bar{\rho} \tilde{T}$, where $R$ is the gas specific constant. The subgrid-scale (SGS) terms, SGS stress tensor $\tau_{i j}^{S}$ and SGS heat flux $q_{j}^{S}$, are modeled as follows:

$$
\tau_{i j}^{S}-\frac{1}{3} \tau_{k k}^{S} \delta_{i j}=-2 \mu_{t}\left(\tilde{S}_{i j}-\frac{1}{3} \tilde{S}_{k k} \delta_{i j}\right), \quad \tau_{k k}^{S}=2 C_{I} \bar{\rho} \Delta^{2}|\tilde{S}|^{2}, \quad q_{j}^{S}=\frac{\mu_{t} \gamma c_{v}}{P r_{t}} \partial_{j} \tilde{T}
$$

where $\mu_{t}=\bar{\rho} C_{s} \Delta^{2}|\tilde{S}|,|\tilde{S}|=\sqrt{2 \tilde{S}_{i j} \tilde{S}_{i j}}$ and $\Delta$ is the filter width. It is remarked that for the current study we use implicit filtering in (7-9), with the filter width determined by the grid spacing. LES with explicit filtering will be considered in future studies.

In the Smagorinsky model $C_{s}$ is defined as a problem-specific constant. In this study we use $C_{s}=0.0085$ [33]. Simulations using this model are denoted as LES1.

In the dynamic SGS model the Smagorinsky constant $C_{S}$ and the constant for the isotropic part of the SGS stress $C_{I}$ are obtained through the Germano-Lilly [34] procedure:

$$
C_{s}=\frac{\left\langle L_{i j}^{C_{s}} M_{i j}^{C_{s}}\right\rangle_{H}}{\left\langle M_{i j}^{C_{s}} M_{i j}^{C_{s}}\right\rangle_{H}}, \quad C_{I}=\frac{\left\langle L_{l l}\right\rangle_{H}}{\left\langle M_{l l}^{C_{I}}\right\rangle_{H}},
$$

where

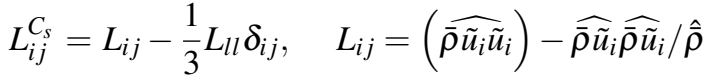

$$
\begin{aligned}
& M_{i j}^{C_{S}}=-2 \hat{\bar{\rho}} \hat{\Delta}^{2}|\hat{\tilde{S}}|^{2}\left(\tilde{S}_{i j}-\frac{1}{3} \tilde{S}_{l l} \delta_{i j}\right)+2 \Delta^{2}\left[\left(\bar{\rho} \mid \widehat{\tilde{S} \mid \tilde{S}_{i j}}\right)-\frac{1}{3}\left(\bar{\rho}|\widetilde{S}| \widetilde{S}_{l l} \delta_{i j}\right)\right] \\
& M_{l l}^{C_{I}}=2 \hat{\bar{\rho}} \hat{\Delta}^{2}|\hat{\tilde{S}}|^{2}-2 \Delta^{2}\left(\widehat{\bar{\rho}|\widetilde{S}|^{2}}\right)
\end{aligned}
$$

and $<f>_{H}$ stands for averaging in homogeneous directions. The Germano procedure requires an explicit filtering operation, denoted here with the top hat symbol. For this filtering operation we use a 3D operator based on a 1D trapezoidal filter:

$$
\hat{f}_{i}=\frac{1}{4} f_{i-1}+\frac{1}{2} f_{i}+\frac{1}{4} f_{i+1}
$$

Simulations using this dynamic SGS model are denoted as LES2.

For the cases with low turbulent Mach number $M_{t}<0.4$ it is shown [33] that the isotropic part of the SGS stress can be neglected $\left(C_{I}=0\right)$. Early numerical experiments comparing the case of setting $C_{I}=0$ vs. the non-zero case produced a similar 
result by the LES1 model for a 3D isotropic turbulence test case using a $M_{t, 0}=0.6$ [9]. There is however, a slightly different result than the LES2 on some of the computed flow quantities by using the non-zero $C_{I}$. Only results using $C_{I}=0$ will be presented for the same isotropic turbulence test case in the later section.

\section{Test Cases}

This section illustrates the performance of our high-order filter scheme for DNS and LES of two 3D low speed turbulence flows considered in [9]. The first test case is the nearly incompressible (inviscid) Taylor-Green vortex problem and its viscous counterpart. The second test case is the decay of an isotropic turbulence with shocklets for an initial turbulent Mach number $M_{t, 0}=0.6$. For both test cases grid convergence studies are performed using uniform $256^{3}, 128^{3}$ and $64^{3}$ grids for the DNS simulations. Grid convergence studies also are performed using uniform $128^{3}, 64^{3}$ and $32^{3}$ grids for LES computations. Studies found that for an accurate numerical dissipation control scheme, a coarse grid DNS using a uniform $64^{3}$ grid compared well with the filtered DNS using a fine grid of $256^{3}$ grid points (spectrally filtered to a $64^{3}$ grid). For the LES computations the $32^{3}$ grid is too coarse for obtaining an accurate solution, whereas, the $128^{3}$ grid solutions are almost on top of the filtered DNS computation on the $256^{3}$ grid. Here, only the results using the $64^{3}$ are shown.

\subsection{Taylor-Green Vortex}

The first test case is the 3D Taylor-Green vortex [35] inviscid flow. This problem has been studied broadly in e.g. $[9,36,12]$ and many others. The 3D Euler equations are solved with gas constant $\gamma=5 / 3$. The computational domain is a $2 \pi$ square cube using a uniform $64^{3}$ grid. Boundary conditions are periodic in all directions.

The initial conditions are

$$
\begin{aligned}
& \rho=1, \quad p=100+([\cos (2 z)+2][\cos (2 x)+\cos (2 y)]-2) / 16, \\
& u_{x}=\sin x \cos y \cos z, \quad u_{y}=-\cos x \sin y \cos z, \quad u_{z}=0 .
\end{aligned}
$$

The initial turbulent Mach number is $M_{t, 0}=0.042$ and the final time is $t=10$. We also consider the viscous counterpart of the Taylor-Green vortex problem. In the viscous case the physical viscosity is assumed to follow a power-law:

$$
\mu / \mu_{\text {ref }}=\left(T / T_{r e f}\right)^{3 / 4} .
$$

Here we use $\mu_{\text {ref }}=0.005$ and $T_{\text {ref }}=1$ in non-dimensional units. The initial Reynolds number is $R e_{0}=2040$. For this low-Mach number flow without high shear regime the simulation actually does not require any numerical dissipation. However, 
we use the same shock-capturing scheme with adaptive numerical dissipation control to demonstrate its accurate performance for such low-Mach number cases. The key study involves the assessment of accuracy of the computed solution using different forms of $\kappa_{j+1 / 2}^{l}$ and different values of $\delta$ mentioned above.

\subsubsection{Inviscid Case - DNS Scheme Comparison}

In the inviscid case the kinetic energy should be constant. It can be used as a criterion to judge the accuracy of the four considered filter numerical fluxes. The coarse grid DNS ( $64^{3}$ grid - no SGS model) comparison among different methods is shown in Fig. 2. Figure 2 shows the temporal evolution of the mean kinetic energy and enstrophy comparing with the $256^{3}$ grid filtered DNS reference solution. The preservation of kinetic energy is achieved with C10-split, WENO9fi-Dsplit-WavD and WENO9fi-Dsplit-Wav $\kappa=10^{-5}$, while WENO9fi-Dsplit-Wav $\kappa(i)$ obtains a small loss in energy after $t \approx 6$. All four methods presented on the enstrophy plot demonstrate good agreement with the semi-analytical solution [36], which is defined on the interval $0 \leq t \leq 3.5$. The enstrophy values obtained using WENO9fi-Dsplit-Wav $\kappa(i)$ are slightly smaller than those obtained using the other three methods.

\subsubsection{Viscous Case - DNS and LES Scheme Comparison}

The temporal evolution of the mean-square velocity and enstrophy of the coarse grid DNS (no SGS model) results on a $64^{3}$ grid by different methods are shown in Fig. 3. The reference solution is the DNS simulation using a $256^{3}$ grid and spectral filtering to the $64^{3}$ grid. For this viscous case the most accurate cut off parameter $\delta$ in WENO9fi-Esplit-WavD and WENO9fi-Dsplit-Ducr is when $\delta=1$. The kinetic energy computed solutions by all considered methods matches the reference solution. The difference between methods is only visible on the enstrophy comparison, though all the results are very close to the reference solution. The methods using Ducros et al. split C10-Dsplit and WENO9fi-Dsplit-Wav $\kappa=10^{-5}$ as well as WENO9fi-Esplit-Wav $\kappa(i)$ obtain slightly more accurate results than C10-Esplit and WENO9fi-Esplit-WavD.

The results obtained using the two LES models are shown in Figs. 4 and 5. As observed also in the isotropic turbulence simulations (to be shown later), results obtained in LES1 are closer to the reference solution than the results obtained using the dynamic model LES2. All LES methods underestimate both the kinetic energy and the enstrophy. WENO9fi-Esplit-Wav $\kappa(i)$ is slightly less accurate than C10Dsplit and WENO9fi-Esplit-WavD. The accuracy by C10-Esplit and C10-Dsplit are almost the same. 

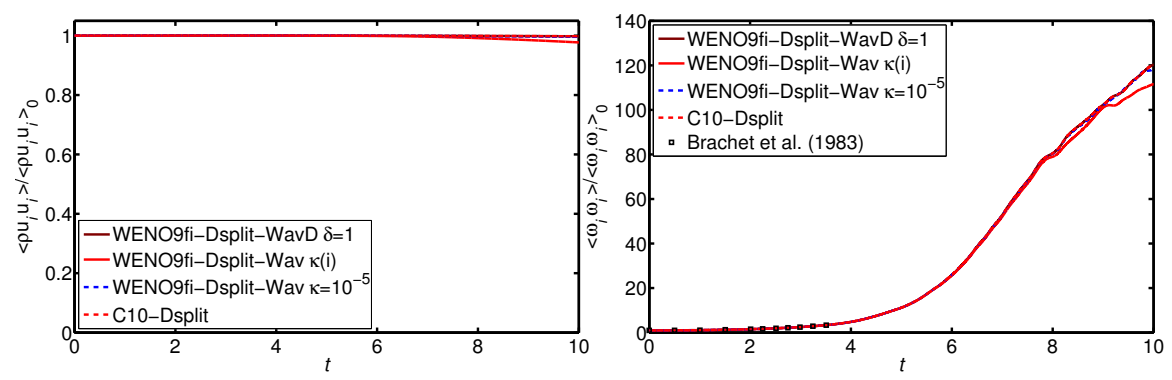

Fig. 2 Coarse Grid DNS scheme comparison for the inviscid Taylor-Green vortex problem using a $64^{3}$ grid: Temporal evolution of the kinetic energy (left) and enstrophy (right).
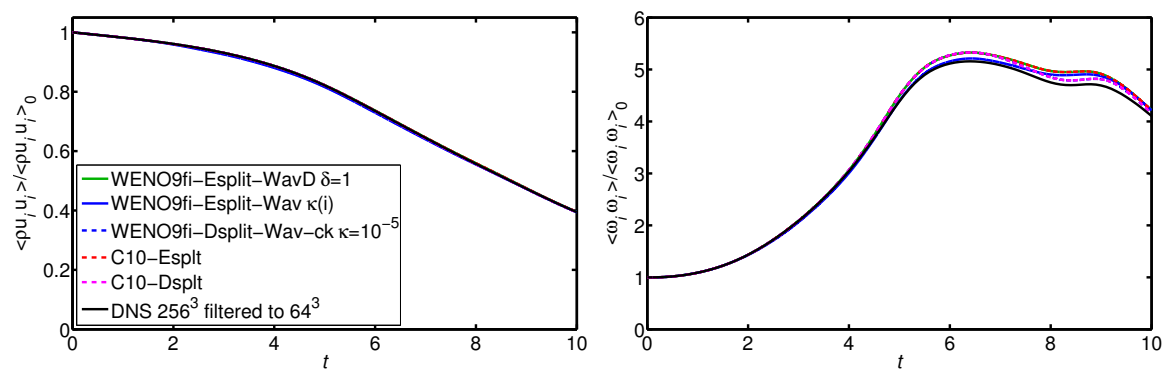

Fig. 3 Coarse Grid DNS scheme comparison for the viscous Taylor-Green vortex problem using a $64^{3}$ grid: Temporal evolution of the kinetic energy (left) and enstrophy (right). The reference solution is the DNS computation on a $256^{3}$ grid and spectrally filtered to a $64^{3}$ grid.
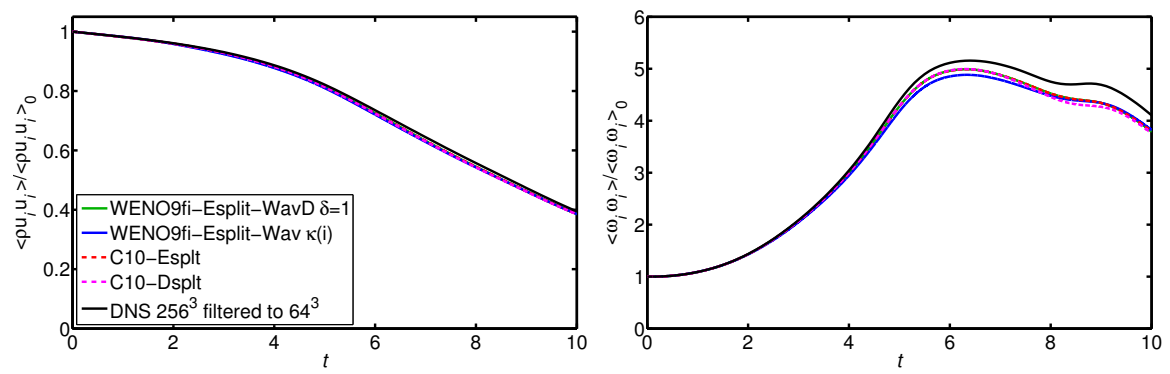

Fig. 4 LES1 scheme comparison for the viscous Taylor-Green vortex problem using a $64^{3}$ grid: Same as Fig. 3 with the results obtained using the Smagorinsky SGS model (LES1). 

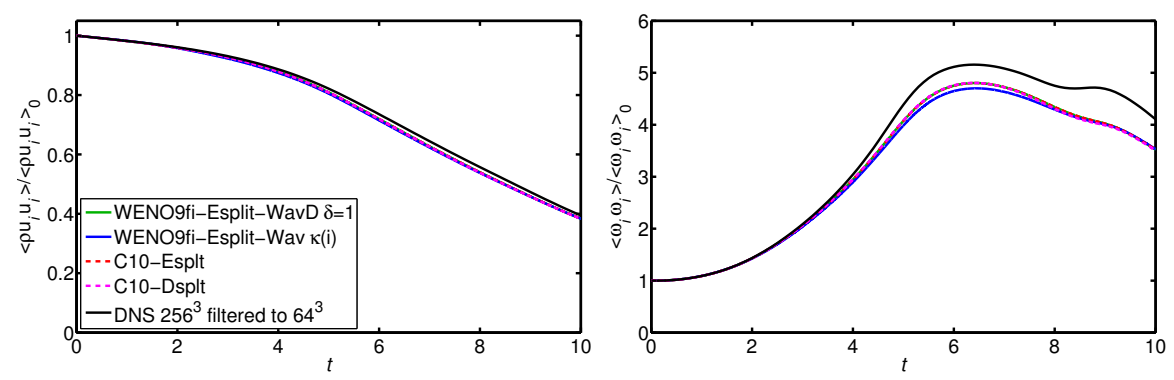

Fig. 5 LES2 scheme comparison for the viscous Taylor-Green vortex problem using a $64^{3}$ grid: Same as Fig. 3 with the results obtained using the Germano-Lilly SGS model (LES2).

\subsection{Compressible Isotropic Turbulence}

The second test case is the decaying compressible isotropic turbulence with eddy shocklets [37, 9], see e.g. [37, 9, 14, 15] For high enough turbulent Mach number, $M_{t}$ weak shock waves (shocklets) develop spontaneously from the turbulent motions. For the current numerical experiment we set the initial $M_{t, 0}=0.6$. The NavierStokes governing equations and the filtered governing equations (7-9) are solved using $\gamma=1.4$. The computational domain is on the $2 \pi^{3}$ cube with periodic boundary conditions in all directions. The physical viscosity is assumed to follow a power-law (18).

The initial condition consists of a random solenoidal velocity field $u_{i, 0}$ that satisfies

$$
E(k) \sim k^{4} \exp \left(-2\left(k / k_{0}\right)^{2}\right), \quad \frac{3}{2} u_{r m s, 0}^{2}=\frac{\left\langle u_{i, 0} u_{i, 0}\right\rangle}{2}=\int_{0}^{\infty} E(k) d k
$$

The brackets here denote averaging over the entire computational domain. For this study we put $u_{r m s, 0}=1$ and $k_{0}=4$. The density and pressure fields are initially constant with initial turbulent Mach number $M_{t, 0}=0.6$ and Taylor-scale Reynolds $R e_{\lambda, 0}=100$. These parameters are defined as follows:

$$
M_{t}=\frac{\sqrt{\left\langle u_{i} u_{i}\right\rangle}}{\langle c\rangle}, \quad R e_{\lambda}=\frac{\langle\rho\rangle u_{r m s} \lambda}{\langle\mu\rangle}, u_{r m s}=\sqrt{\frac{\left\langle u_{i} u_{i}\right\rangle}{3}}, \lambda=\sqrt{\frac{\left\langle u_{x}^{2}\right\rangle}{\left\langle\left(\partial_{x} u_{x}\right)^{2}\right\rangle}} .
$$

The time scale is $\tau=\lambda_{0} / u_{r m s, 0}$ and the final time is $t / \tau=4$. The final turbulent Mach number is $M_{t}=0.29$.

Similar to the Taylor-Green vortex problem, different values of $\kappa$ and $\delta$ parameters are examined. Unlike the Taylor-Green vortex case, the most accurate solutions are obtained using a smaller $\kappa$ and for vales of $\delta$ between 0.7 and 1 .

Figure 6 shows the $64^{3}$ coarse grid comparison of three high order methods. They compare well with the $256^{3}$ DNS at low $k$ value. Comparisons of the temporal evolutions of the mean-square velocity, enstrophy, temperature variance and dilatation using by the various filter numerical fluxes on a $64^{3}$ coarse grid DNS 
(no SGS model) are shown in Fig. 7. The reference solution was obtained from the DNS simulation using a $256^{3}$ grid and spectral filtering to a $64^{3}$ grid (digitized from [9]). The best results are obtained with C10-AV12, WENO9fi-Dsplit-Wav $\kappa(i)$ and WENO9f-Esplit-Ducr. The cut-off parameter of the Ducros et al. sensor in WENO9fi-Esplit-WavD is $\delta=0.7$. However, the results remain almost the same when $\delta$ increases slightly beyond 0.7 . For the dilatation, the best match with the reference solution is obtained by method C10-AV12. However, this scheme underestimates the enstrophy, while the rest of the methods either match or slightly overestimate the enstrophy.

Figure 8 shows the $64^{3}$ grid LES1 comparison of three high order methods. They compare well with the $256^{3}$ DNS solution. Figure 8 shows the comparison of the $64^{3}$ coarse grid comparison of three high order methods. They compare well with the $256^{3}$ DNS at low $k$ value. The results obtained using the two LES models are shown in Figs. 9 and 10. The LES1 computations are closer to the reference solution than the dynamic model LES2. The best results in both cases are obtained with C10Esplit, WENO9fi-Esplit-Ducr and WENO9fi-Esplit-WavD. All presented methods in LES1 and LES2 underestimate the enstrophy and kinetic energy. The spectra of this isotropic decaying turbulence test case were examined, the computed spectra by these schemes are as expected and results are not shown due to a space limitation.

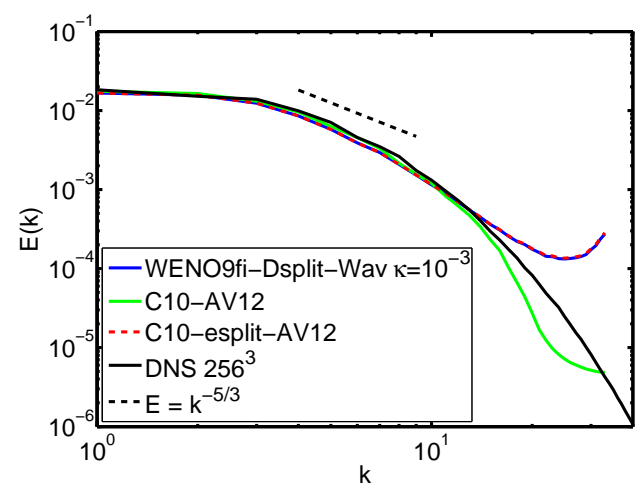

Fig. 6 Spectra of coarse grid DNS scheme comparison for isotropic turbulence problem using a $64^{3}$ grid.

\section{Conclusions}

The performance of our high order nonlinear filter scheme with different flow sensors has been demonstrated in LES and DNS of low-Mach number flows. The forms (1) - (4) for the filter numerical flux were chosen to demonstrate that for low speed 

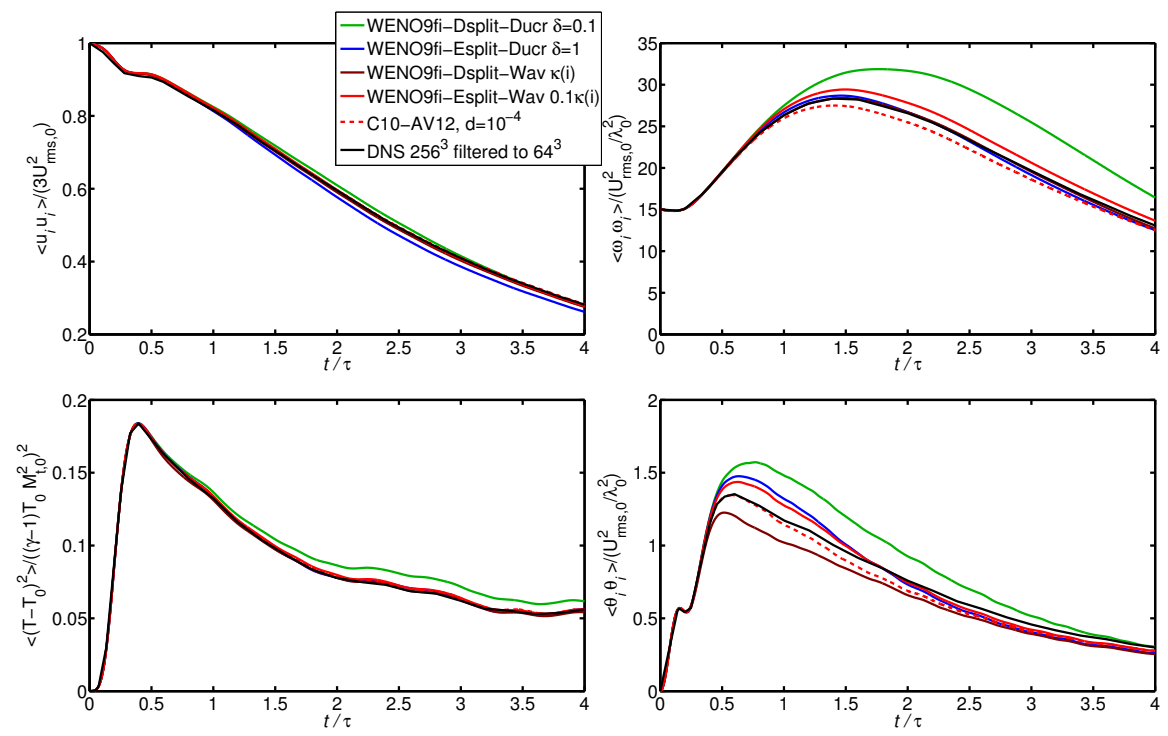

Fig. 7 Coarse grid DNS scheme comparison for the isotropic turbulence problem using a $64^{3}$ grid: Temporal evolution of kinetic energy (top left), enstrophy (top right), temperature variance (bottom left) and dilatation, $\theta_{i}=\partial_{i} u_{i}$ (bottom right). The reference is the digitized solution from [9] on a $256^{3}$ grid spectrally filtered to a $64^{3}$ grid.

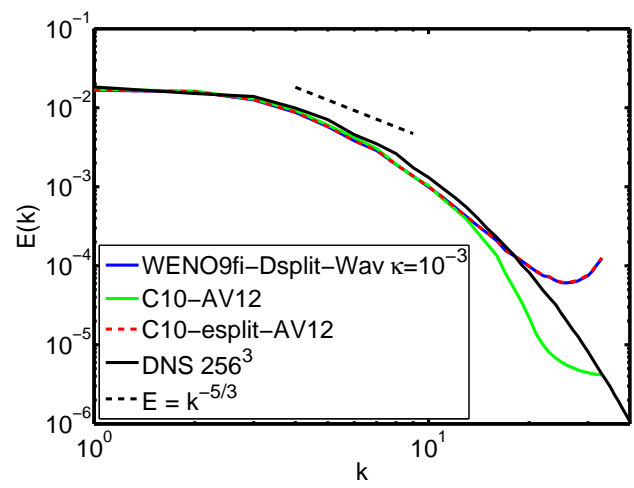

Fig. 8 Spectra of LES1 scheme comparison for isotropic turbulence problem using a $64^{3}$ grid. 

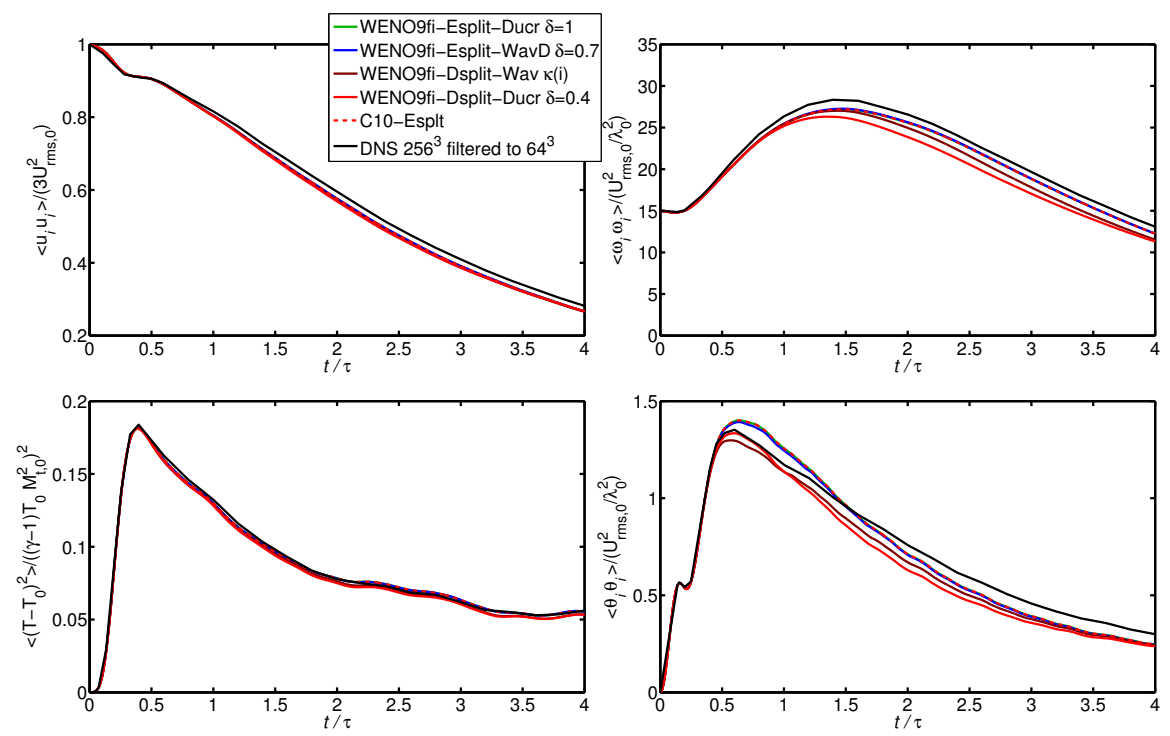

Fig. 9 LES1 scheme comparison for the isotropic turbulence problem using a $64^{3}$ grid: Same as Fig. 7 with the results obtained using the Smagorinsky SGS model (LES1).
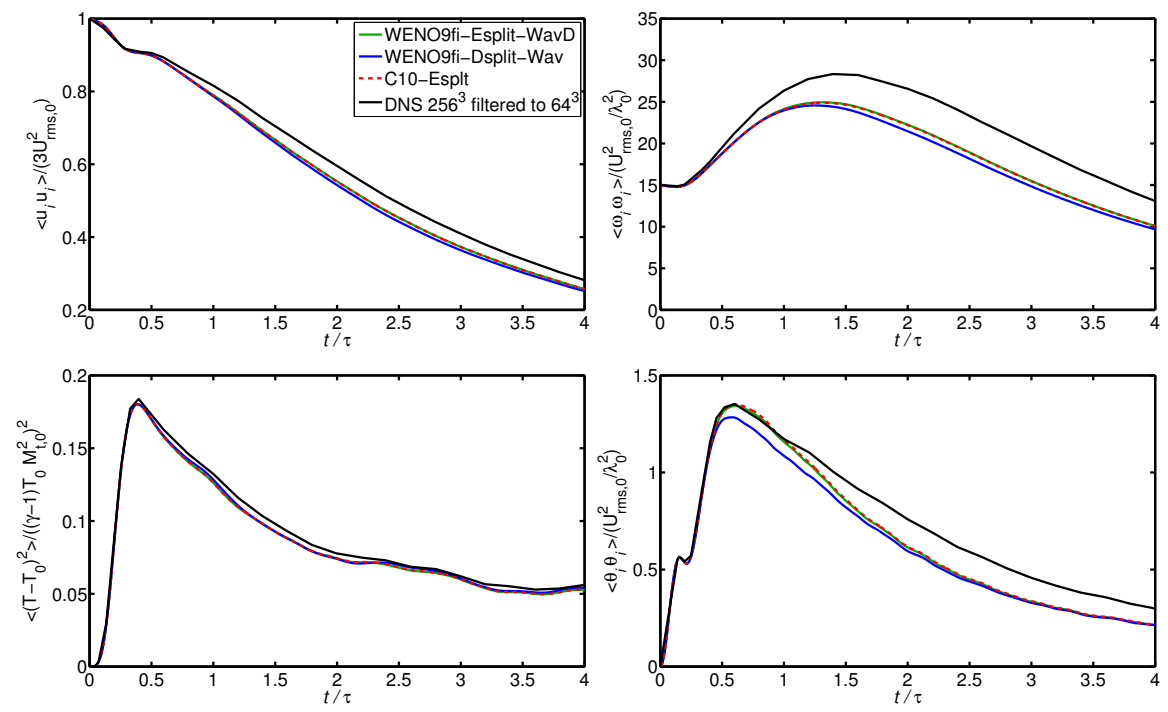

Fig. 10 LES2 scheme comparison for the isotropic turbulence problem using a $64^{3}$ grid: Same as Fig. 7 with the results obtained using the Germano-Lilly SGS model (LES2). 
turbulence flows without strong shear waves, the constant $\kappa$ vs. the local $\kappa_{j+1 / 2}^{l}$ behave similarly. The main difference when using the constant $\kappa$ parameter is that one has to know the flow structure of the entire evolution a priori in order to select the proper constant $\kappa$ parameter. Contrary to the considered low speed flow test cases, our previous investigations $[4,30,3,31,5,32,1,6]$ for various complex high speed shock-turbulence interaction flows, employing the local $\kappa_{j+1 / 2}^{l}$ would provide an automatic selection of the amount of numerical dissipation needed at each flow location, thus, leading to a more accurate DNS and LES simulation with less tuning of parameters. The local flow sensors for high order nonlinear filter schemes are particularly important for flows with a time varying random forcing and a wide range of flow speed regime during time-accurate evolution. See the Appendix for a preliminary simulation of DNS of a strong compressible turbulence with a wide range of shock speeds with time varying random forcing. The complete work is forthcoming for another important area of DNS and LES computations.

Overall, the LES1 model (Smagorinsky) computations are closer to the filtered DNS reference solution than the LES2 model (dynamic Germano). For the isotropic turbulence with shocklets test case, results using non-zero $C_{I}$ formula indicated in (9) vs. setting $C_{I}=0$ are similar using the LES1 model. This behavior might be due to the fact that the final turbulent Mach number for the considered time integration is $M_{t}=0.29$. There is, however, a slightly different result by the LES2 on some of the computed flow quantities by using the non-zero $C_{I}$. Only results using $C_{I}=0$ have been presented in this work. Further investigations on the reason why the computed result by LES1 perform better than by the LES2 model, and the behavior on the choice of the two $C_{I}$ options are needed.

Acknowledgements The support of the DOE/SciDAC SAP grant DE-AI02-06ER25796 is acknowledged. Financial support from the NASA Aerosciences Project - RCA for the second author is gratefully acknowledged. Work by the fourth author was performed under the auspices of the U.S. Department of Energy at Lawrence Livermore National Laboratory under Contract DE-AC5207NA27344. Work by the fifths author was support in part by the NSF grant AST-1412271 and UC Santa Cruz sub award A16-0243-S001

\section{Appendix}

Work in Progress for a Different Flow Type: Understanding 2D Compressible Turbulence with Time-dependent Random Forcing by Low Dissipative High Order Schemes

Problem and Challenges. Over the past few decades DNS of turbulent flows played an ever increasing role in pushing the edge in numerical versus experimental comparisons $[38,39]$. The Reynolds numbers achieved in the largest DNS are of the order $10^{6-7}$, i.e., comparable to the largest Reynolds numbers in laboratory experiments. DNS became a vital tool for addressing basic questions, such as universality in the sense of Kolmogorov's theory, and the dependence of the statistics on the 
Reynolds number and scale. Extreme-resolution DNS will provide high-quality data that are impossible to obtain experimentally. Such DNS are essential to understand intermittency and develop predictive LES of turbulent flows.

It is well known that introduction of even modest compressibility is sufficient to generate small scales through wave steepening, enabling disparate-scale interaction, see e.g. [40]. Besides purely engineering applications, compressible turbulence plays an very important role in a broad range of applications in astrophysics and geophysics (galaxy formation, star formation, supernova explosions, cosmic ray acceleration, black hole accretion, planet formation, solar wind heating, etc.), and in atmospheric sciences (weather prediction and climate modeling). Each of these subjects can potentially receive a strong transformative impact from compressible DNS/LES at the exascale. Recent advances in theory of compressible turbulence have provided important reference points for validation of numerical solutions, ensuring that the flops would not be wasted [41, 42, 43, 44].

Simulation of compressible media is challenging due to conflicting requirements for numerical methods to be accurate enough to resolve the small scales of turbulence, but robust enough to handle shock waves without generating spurious numerical noise during the entire time-accurate evolution.

Preliminary Results and Significance. Figures 11 and 12 illustrate performance of the WENO7fi scheme with a basic example of 2D compressible turbulence. The simulations use a doubly periodic square domain with the flow fully determined by three parameters: grid resolution $N$, energy injection rate $\varepsilon_{f}$, and energy injection scale $\lambda_{f}$. The solenoidal forcing is $\delta$-correlated in time. Comparison of a 2 nd-order scheme PPM [45] with our 7th-order scheme WENO7fi in simulations with grid resolution from $N=2,048$ to 16,384 indicates that the spectral bandwidth of WENO7fi is slightly more than two times larger than that of PPM. This means that the cost of a 2D simulation with WENO7fi, resolving the turbulence to a given degree, is approximately 4 times cheaper computationally than with PPM. For a 3D simulation, the advantage of WENO7fi over PPM would be even more impressive. The key advantage of the adaptive flow sensor in high order methods is that no a priori knowledge of the flow structure is required, even for a mixture of compressible shock-free turbulence, low speed turbulence with shocklets and turbulence with strong shocks, as the system evolves in time. This recent development is expected to provide an improved predictability and reliability of CFD turbulent computations containing both low speed and high speed regimes that can be compromised by standard high order shock-capturing schemes without proper control of numerical dissipation. With the advent of exascale computing and the proper CFD tools, improved understanding of compressible turbulence in a wide range of Mach numbers will soon be within our reach. 


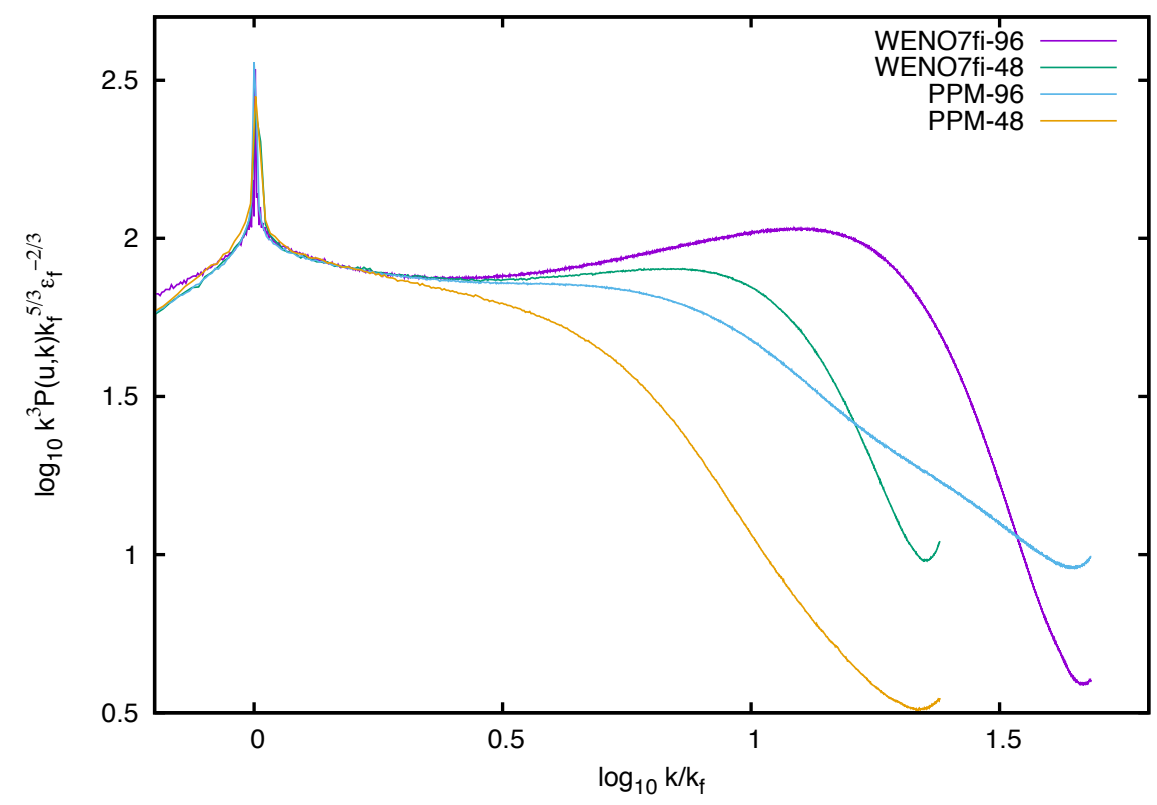

Fig. 11 Compensated velocity power spectra from four simulations of 2D compressible turbulence with the PPM and WENO7fi methods. Only scales below the forcing scale $\lambda_{f}=2 \pi / k_{f}$ resolved with 48 or 96 grid points are shown. 

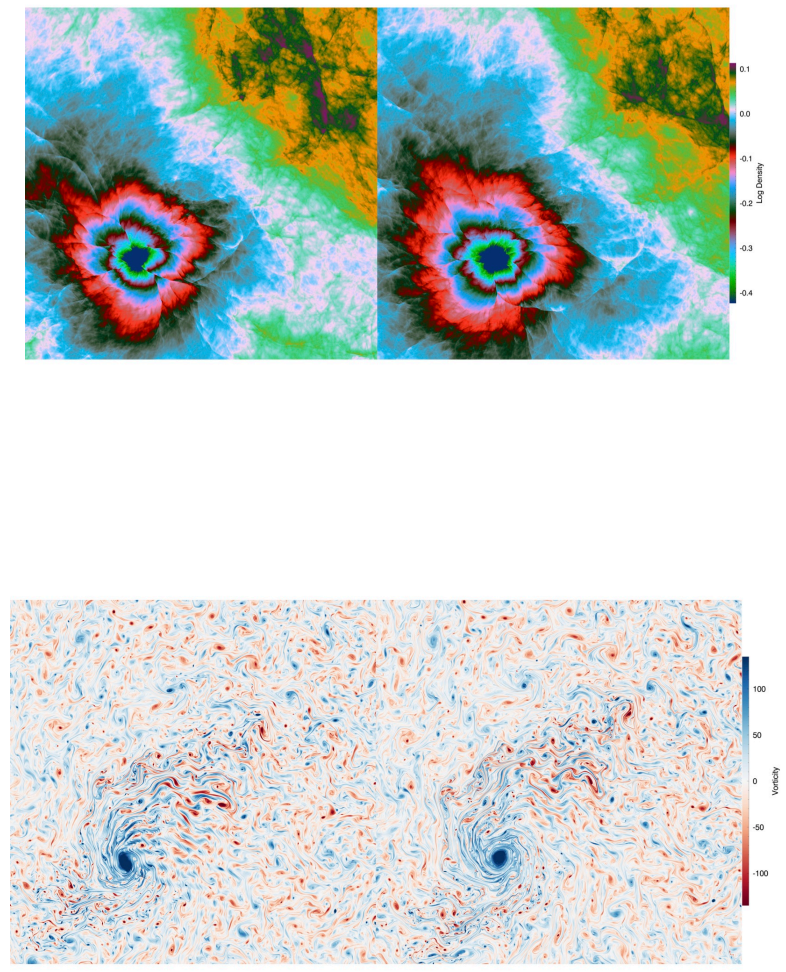

Fig. 12 Density (Top 2 subfigures) and vorticity (Bottom 2 subfigures) fields for sub-volumes of PPM (left) and WENO7fi (right) simulations, respectively, resolving the forcing scale with 96 grid points.

\section{References}

1. H.C. Yee, B. Sjögreen, in Proc. of ICOSAHOM 09 (Trondheim, Norway, 2013)

2. H.C. Yee, B. Sjögreen, J. Comput. Phys. 225, 910 (2007)

3. B. Sjögreen, H.C. Yee, J. Sci. Comput. 20, 211 (2004)

4. H.C. Yee, N. Sandham, M. Djomehri, J. Comput. Phys. 150, 199 (1999)

5. H.C. Yee, B. Sjögreen, A. Hadjadj, Commun. Comput. Phys. 12, 1603 (2012)

6. D. Kotov, H.C. Yee, B. Sjögreen, in Proceedings of the ASTRONUM-2013 (Biarritz, France, 2013)

7. D. Kotov, H.C. Yee, A. Hadjadj, A. Wray, B. Sjögreen, in Proceedings of ICCFD8 (Chengdu, Sichuan, China; also expanded verion submitted to CiCP, 2014, 2014)

8. M. Lombardini, D.J. Hill, D.I. Pullin, D.I. Meiron, J. Fluid Mech. 670, 439 (2011) 
9. E. Johnsen, J. Larsson, A. Bhagatwala, W. Cabot, P. Moin, B. Olson, P. Rawat, S. Shankar, B. Sjögreen, H. Yee, X. Zhong, S. Lele, J. Comput. Phys. 229, 1213 (2010)

10. E. Touber, N. Sandham, Shock Waves 19(6), 469 (2011)

11. S.C. Lo, G. Blaisdell, A. Lyrintzis, J. for Num. Meth. in Fluids 62(5), 473 (2010)

12. D. Drikakis, C. Fureby, F. Grinstein, D. Youngs, J. Turbul. 8(20) (2007). DOI 10.1080/14685240701250289

13. B. Thornber, A. Mosedale, D. Drikakis, J. Comput. Phys. 226, 1902 (2007)

14. B. Thornber, A. Mosedale, D. Drikakis, D. Youngs, R. Williams, J. Comput. Phys. 227, 4873 (2008)

15. D. Drikakis, M. Hahn, A. Mosedale, B. Thornber, Phil. Trans. R. Soc. A 367, 2985 (2009)

16. F. Ducros, V. Ferrand, F. Nicoud, C. Weber, D. Darracq, C. Gacherieu, T. Poinsot, J. Comput. Phys. 152, 517 (1999)

17. H.C. Yee, B. Sjögreen, A. Hadjadj, in Proc. of ASTRONUM-2010 (San Diego, Calif., 2009)

18. P. Olsson, J. Oliger, Energy and maximum norm estimates for nonlinear conservation laws. Tech. Rep. 94.01, RIACS (1994)

19. H.C. Yee, M. Vinokur, M. Djomehri, J. Comput. Phys. 162, 33 (2000)

20. H. Yee, B. Sjögreen, in Turbulent Flow Computation, ed. by D.D..B. Geurts (Kluwer Academic, 2002)

21. F. Ducros, F. Laporte, T. Soulères, V. Guinot, P. Moinat, B. Caruelle, J. Comp. Phys. 161, 114 (2000)

22. B. Sjögreen, H.C. Yee, in Proc. of the 8th Euro. Conf. on Numerical Mathematics \& Advanced Applications (ENUMATH 2009) (Uppsala University, Uppsala, Sweden, 2009)

23. P. Olsson, Math. Comp. 64, 1035 (1995)

24. B. Sjögreen, H.C. Yee, in Proceedings of the Turbulence and Shear Flow Phenomena 5 (TSFP5) (Munich, Germany, 2007)

25. B. Sjögreen, H.C. Yee, M. Vinokur, J. Comput. Phys. 265, 211 (2014)

26. M. Ciment, Leventhal, Math. Comp. 29, 985 (1975)

27. X.S. Li, C.W. Gu, J. Comput. Phys. 227, 5144 (2008)

28. A. Hadjadj, A. Kudryavtsev, J. Turbul. 6, 33 (2005)

29. G.P. M. Farge, K. Schneider, Phys. Rev. Lett. 5, 45011 (2001)

30. N.D. Sandham, Q. Li, H.C. Yee, J. Comp. Phys. 178, 307 (2002)

31. H.C. Yee, B. Sjögreen, Shock Waves 17, 185 (2007)

32. A. Hadjadj, H.C. Yee, B. Sjögreen, Int. J. for Num. Meth. in Fluids 70, 1405 (2012)

33. G. Erlebacher, M.Y. Hussaini, C.G. Speziale, T.A. Zang, J. Fluid Mech. 238, 155 (1992)

34. D.K. Lilly, Physics of Fluids 4(3), 633 (1992)

35. G. Taylor, A. Green, Proc. R. Soc. London A 158, 499 (1937)

36. M. Brachet, D. Meiron, S. Orszag, B. Nickel, R. Morf, U. Frisch, J. Fluid Mech. 130, 411 (1983)

37. S. Lee, S. Lele, P. Moin, Phys. Fluids 3, 657 (1991)

38. T. Ishihara, T. Gotoh, Y. Kaneda, Annu. Rev. Fluid Mech. 41, 165 (2009)

39. R. Benzi, L. Biferale, J. Stat. Phys. (2015)

40. P.H. Diamond, S.I. Itoh, K. Itoh, Modern Plasma Physics (2010)

41. S. Galtier, S. Banerjee, Phys. Rev. Lett. 107(13), 134501 (2011)

42. S. Banerjee, S. Galtier, Phys. Rev. E 87(1), 013019 (2013)

43. H. Aluie, Physica D 247, 54 (2013)

44. A.G. Kritsuk, R. Wagner, M.L. Norman, J. Fluid Mech. 729, R1 (2013)

45. P. Colella, P.R. Woodward, J. Comput. Phys. 54, 174 (1984) 